\title{
The Grating Data Collection System Based on Dynamic Link Library and Its Application in Engineering Control
}

\author{
Zhonghui Luo ${ }^{1, a}$, Qijun Xiao ${ }^{2, b}$, * \\ ${ }^{1}$ Department of Mechanical and Electronic Engineering, Guangdong Polytechnic Normal University, \\ Guangzhou, 510635, China \\ ${ }^{2}$ Faculty of Electronic Information and Mechanical \& Electrical Engineering, Zhaoqing University, \\ Zhaoqing, 526061, China \\ aemail: Lzh2382002@163.com, bemail: Shawqj@126.com, " corresponding author
}

Keywords: The Dynamic Link Library, Grating, Hydraulic Servo Control, Data Acquisition System

\begin{abstract}
In the precision electro-hydraulic servo control system, grating is commonly used as displacement detecting element. To realize getting the data from the grating displacement sensor and send a command word rapidly, the dynamic link library (DLL) technology is used in this paper, it is that a dynamic link library designed in the Visual C++ (VC) and be call by Visual Basic (VB), thus to achieve rapid data transmission and reduce the difficulty of application of VC programming. Research shows that adopting this technology design can realize fast data acquisition with simple structure and fast data transmission, which can satisfy the precision requirement of the real-time control.
\end{abstract}

\section{Introduction}

With the continuous development of the computer measurement and control technology, the computer needs to collect the sensor signal to be analyzed, calculated and outputted to control field device. The sensor most widely used in the electro-hydraulic servo system is grating displacement sensor being used to detect the hydraulic cylinder position displacement. The signal acquisition interface card can be used for grating, and digital display instrument can also be used. Due to the digital display instrument need communicate with the computer through RS232 serial port to send signals to the computer, the time of transmission need to be considered, therefore ,it is not suitable for high real-time requirements of control system. And the use of interface card has fast conversion speed without data transmission, it need only reading of the specified address to get BCD code pulse signal, the displacement digital signal can be obtained after numerical transformation.

\section{DLL Technologies by VB}

VB is a kind of visualization, object-oriented, event-driven structured high-level programming language under Windows operating system ,due to its characteristic of easy to learn, graphical interface, convenient operation, powerful function, and get back to the user's favor, but VB lack of input output function, API also do not provide the input and output functions for port, in a hydraulic servo experiment system, it is needed to acquire the data from the grating displacement sensor and send a command word, all need to port input and output operation. In order to solve the above problems, we can take advantage of the DLL technique, namely to create a DLL in the VC, using the DLL in V b has the function of port input and output, so that to greatly reduce the difficulty of the application of VC programming, thus to improve the efficiency, to make the modification of application more simple and convenient, we can write all sorts of DLL function to form various sorts of clear structure, easy modification and convenient application function. Because the VC under WINDOWS has port input output function, therefore, we can make use of VC language to create a DLL with special function.

How to use the VC language to create DLL is that to build a new project to be compiled under 
the environment of VC environment to generate DLL file for VB call. DLL file is stored under in DEBUG directory. Then copy the DLL files to WIND OWS $\backslash$ SYSTEM directory, VB will search the file in the default WINDOWS $\backslash$ SYSTEM directory. First we add a Declare statement. How to use under VB DLL file is that firstly we need to add a Declare statement in the code window general. If the process returns a value, it will be a stated as the Function, if no value returned, it can be declared as Sub, so the DLL can be called.

\section{Data Acquisition System Design}

High precision grating displacement sensor and sampling interface card are used for hardware and VB software is used in the design for data acquisition program, the data formats should be firstly needed understood for the program design to familiar with data processing.

Hardware construction This test system is mainly composed of industrial personal computer, four channel high-speed sampling grating sensor interface card SDC-2, grating sensor, and hydraulic cylinder, the industrial personal computer is used to collect the hydraulic cylinder piston displacement data from data acquisition interface card to realize data storage, data analysis, curve drawing, data report, and print function. Grating ruler use the KA-30120 type linear grating ruler,which is mainly composed of the ruler body and reading head. The pitch spacing is $0.001 \mathrm{~mm}$ named $1000 \mathrm{line} / \mathrm{m})$, the precision can reach $l \mu \mathrm{m}$, and the range is $0-300 \mathrm{~mm}$, SDC- 2 card is specially designed for grating sensor signal acquisition, each channel sampling frequency can reach as high as $2.5 \mathrm{MHz}$, if using $0.001 \mathrm{~mm}$ resolution grating ruler, the sampling frequency can reach above $120 \mathrm{~m} / \mathrm{min}$. This card makes various relevant detection convenient for $\mathrm{l}$ development and production of control product, it can meet the demand of teaching experiment, scientific research, etc, the technical characteristics is four channel grating data processing, which can be used for the linear encoder data acquisition with high sampling frequency of $2.5 \mathrm{MHz}$, ISA bus interface and grating ruler reference point coordinates reset function for memory and recovery for datum point, address selection of FO uses jumper which can choose address range from $200 \mathrm{H}$ to $3 \mathrm{~F} 0 \mathrm{H}$, the default address of manufacturer is $320 \mathrm{H}$. Data string begins with $\mathrm{F} 8 \mathrm{H}$, and end with F9H.Among them, $\mathrm{X}, \mathrm{Y}, \mathrm{Z}, \mathrm{W}$ on behalf of $\mathrm{X}$ axis, $\mathrm{Y}$ axis, $\mathrm{Z}, \mathrm{Z}$ axis, $\mathrm{W}$ axis respectively. $\mathrm{J}$ means the axis is usually lie in signal acquisition and display status, $\mathrm{F}$ means the axis in the grating ruler reference point search status. $\mathrm{H}$ means the axis has already been reset for grating ruler reference point coordinates.

Software development Data acquisition software send command word to address HEX320, and then read the port, after delay for a period of time to read four channel sampling data from zero to nineteen with total of 20 bytes, $0,5,10,15$ bytes is flag state data, when BIT0 equal 1, it represents for negative sign, instead of positive sign, when BIT1 equal 1, it is on behalf of the $\mathrm{x}$ axis that is in a state of "F", when BIT2 equal 1,it is on behalf of the axis that is in a state of "H", when BIT4 equal 1,it is on behalf of the state of the axis that has been opened for the state of "G", four bytes behind on behalf of 8 bits BCD pulse number. By changing the delay time procedure to change the sampling frequency, PC acquires data in the appropriate sampling frequency. From the data acquisition card reading program flow shown in Fig.1. The part of the program for original data acquisition is as follows:

Private Declare Function inport Lib “port. dll” as integer

Private Declare Sub OUTPOET Lib "port. dll” Alias

"outport”(By Val ported as integer, By Val nbyte as integer)

const pio $=\& H 320$

Data=import(pio)

Do While (Data $<>\&$ HF8 )

For $\mathrm{k}=0$ to 200000

Next

Data=import(pio)

Loop

For $\mathrm{i}=1$ to 20 
For $\mathrm{k}=1$ to 2000

Next

Data=inport(pio)

While(Data $=\&$ HFF or Data=\&HF8)

Data=import(pio)

End

$\mathrm{a}(\mathrm{i})=$ Data

If(a(i)=\&HF9) Then

Exit For

End if

Next

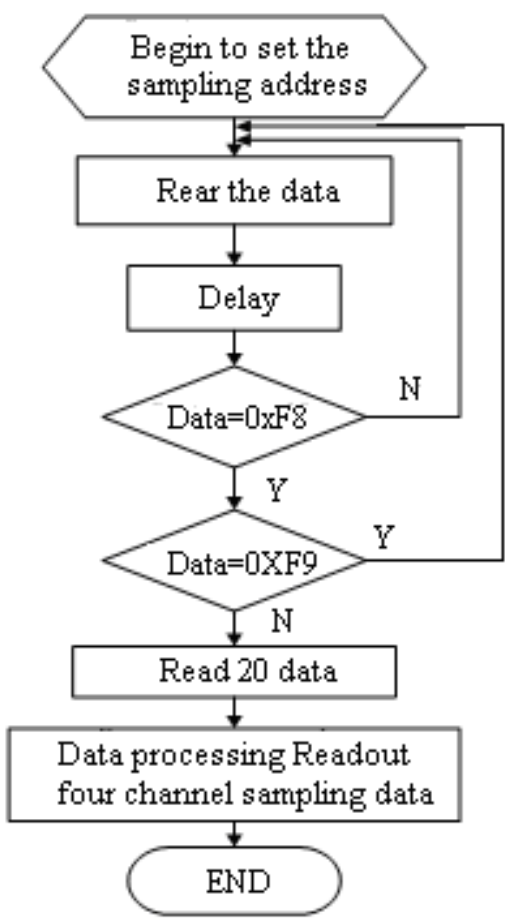

Fig.1. Data acquisition card reading program flow

The collected data array a after dealing with to form four channel pulse digital signal, after multiplied by the lattice spacing of displacement signal to obtain the displacement signal. Through output command word to address HEX320 can also perform reset, display, search, and other functions for axis of the grating.

Test interface can be divided into curve drawing, data processing and file storage parts. The displacement curve drawing is drawn in Pictuerbox first with drawing reference $\mathrm{x}$-coordinate for sampling points and x-coordinate for displacement besides labeling and scale. Using Timer control in to draw real-time displacement curve inside Picture box drawing picture, using VB Line command to draw curves, when curve runs to the right boundary of coordinate, screen is clear to redraw grid line and draw the curve from left to right in the form of transformation of coordinates, at the same time, the Sampling data can be stored in ACCES database or text file, and MALAB can be called to do data analysis processing with data and graphics file print function, as well as the interface is good with simple procedures.

\section{Experiment Research}

Closed-loop control system of the system hardware experiment mainly consists of industrial controlling computer, grating displacement sensor, grating sensor high-speed sampling interface card, D/A card, proportional controller, cylinder and proportional direction valve. Its composition structure is shown in Figure 2. The control principle is: the industrial personal computer receives 
the feedback signal from the grating displacement sensor; the signal is output through the controller to control the aperture and direction of the electro-hydraulic proportional valve, and thus controlling the displacement and speed of the hydraulic cylinder.

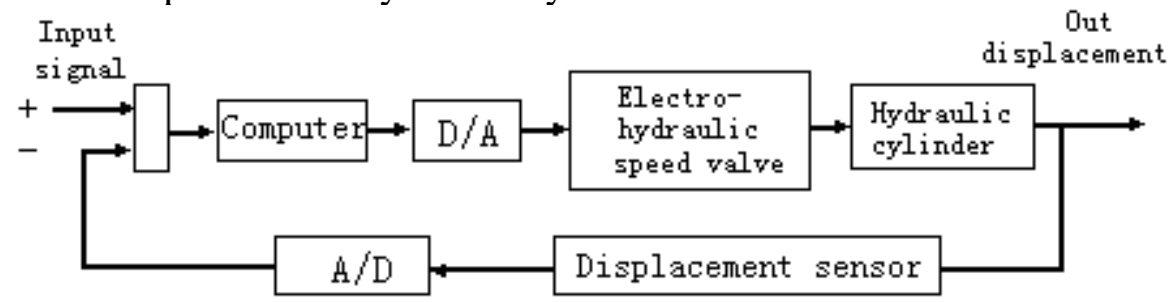

Fig.2. The structure of simulation experiment system

The result of step experiment loading with $200 \mathrm{~kg}$ is following: the step signal is $100 \mathrm{~mm}$, steady-state error of this control system is $0.49 \mathrm{~mm}$, and the response time is $4.23 \mathrm{~s}$. The experimental results satisfy the requirements of practical engineering.

\section{Conclusion}

Sampling interface card can satisfy the requirement of high precision grating sensor test. By using DLL to improve the function of VB, and it is widely used in data collection, DIA card also need to complete the given operation with the call the DLL , and it has powerful functions, and it is an effective way to solve the VB defects. In the future design of data acquisition program, VB, with its concise style will get more extensive application.

\section{Acknowledgement}

This work was supported by Guangzhou science and technology and Information Bureau(No. 201510010235), the Natural Science Foundation of Guangdong province(No. 2015A030313666), and the National Natural Science Foundation of China (No. 11174299).

\section{References}

[1] Zhu Huiqiao and so on. Blend programming with $\mathrm{C}++$ and visual basic by using dynamic link library [J]. Information Technology, 2010 (12)135-139.

[2] Gao Liansheng and so on. Application of dynamic link library in SCADA / HMI [J]. Industrial Control Computer, 2010: 23(6)21-22.

[3] Li Juan and so on. Mixed programming of Visual C++ based on dynamic link library [J]. Computer Engineering and Design, 2010: 31(9):2140-2143

[4] Xu Guoquan and so on. Applications of fiber bragg grating sensing technology in engineering [J]. chinese optics, 2013: 6(3)306-315.

[5] Wang Xiaodong and so on. Techniques of data acquisition and processing in fiber grating sensing system [J]. Instrument Technique and Sensor, 2008 (5)47-51.

[6] ROBERTO M, S IMONE P. A temperature-compensated rotational position sensor based on fiber bragg gratings [J]. Sensors and Actuators A: Physical, 2006: 32( 2 ) 533- 540.

[7] CHALAPATI M T, PATTNA IK P K, SELVARAJAN A, et a.l A Method to discriminate strain and temperature in fiber bragg grating sensors [J]. In Proceedings SICon /05-Sensors for Industry Conference, Houston, Texas, USA, 2005.

[8]Wu An. Development and application of data acquisition software for fiber bragg grating sensing system[M]. Wuhan University of Technology Press, 2009. 